Portland State University

PDXScholar

Portland Institute for Computational Science

Publications

Portland Institute for Computational Science

$6-2018$

\title{
Cox Processes for Counting by Detection
}

Purnima Rajan

Johns Hopkins University, purnima.v.rajan@gmail.com

Yongming Ma

Portland State University, yongming@pdx.edu

Bruno Jedynak

Portland State University, bruno.jedynak@pdx.edu

Follow this and additional works at: https://pdxscholar.library.pdx.edu/pics_pub

Part of the Mathematics Commons

Let us know how access to this document benefits you.

Citation Details

published as: Rajan, P., Ma, Y. \& Jedynak, B. J Math Imaging Vis (2019) 61: 380. https://doi.org/10.1007/ s10851-018-0838-5

This Post-Print is brought to you for free and open access. It has been accepted for inclusion in Portland Institute for Computational Science Publications by an authorized administrator of PDXScholar. Please contact us if we can make this document more accessible: pdxscholar@pdx.edu. 


\title{
Cox Processes for Counting by Detection.
}

\author{
Purnima Rajan · Yongming Ma · Bruno Jedynak
}

Received: date / Accepted: date

\begin{abstract}
In this work, Cox processes and Convolutional Neural Net classifiers (CNNs) are used to estimate the number of instances of an object in an image. Poisson processes are well suited to model events that occur randomly in space, such as the location of objects in an image or the enumeration of objects in a scene. Doubly Stochastic Poisson (or Cox) processes offer increased flexibility, but their computational complexity and storage requirements do not easily scale with image size, typically requiring $O\left(n^{3}\right)$ computation time and $O\left(n^{2}\right)$ storage, where $n$ is the number of pixels in an image. To mitigate this problem we employ the Kronecker algebra, which takes advantage of direct product structures. As the likelihood is non-Gaussian, the Laplace approximation is used for inference, employing the conjugate gradient and Newton's method. Our approach has then close to linear performance, requiring only $O\left(n^{3 / 2}\right)$ computation time and $O(n)$ memory. The proposed algorithm consists of selecting a subset of bounding boxes in the image domain, and querying them for the presence of the object of interest by running a pre-trained CNN classifier. The resulting observations are then aggregated and a posterior distribution
\end{abstract}

P. Rajan

Johns Hopkins University

3400 N Charles St, Baltimore, MD-21218, USA.

Tel.: +1443-692-7768

E-mail: purnima@cs.jhu.edu

Y. Ma

Portland State University

724 SW Harrison St Portland, OR 97201, USA.

E-mail: yongming@pdx.edu

B. Jedynak

Portland State University

724 SW Harrison St Portland, OR 97201, USA.

E-mail: bjedyna2@pdx.edu over the intensity of a Cox process is computed. This intensity function is summed-up, providing an estimator of the number of instances of the object over the entire image. Results are presented on simulated data and on images from the publicly available MS COCO dataset. We compare our counting results with the state-of-theart detection method, Faster $R C N N$, and demonstrate superior performance.

Keywords Object Counting, Gaussian process, Cox processes, Computer Vision, Bayesian inference.

\section{Introduction}

The task of counting objects of interest appears in numerous applications, such as estimating the number of vesicles or mitochondria in electron microscopy [29], counting the number of animals in aerial images for wildlife conservation [1], monitoring humans in surveillance videos [25] [3], or for evaluating astronomical data [49]. Even in applications where enumeration is not the end goal, the object counts provide a high level feature which could help better understand a scene. Moreover, counting over sub-images allows for shallow localization and even precise localization when the sub-images provide a fine-grained partition of the original image.This work proposes to use object detection as the basis for estimating counts. Faster RCNN [38], is among the best algorithms for object detection on popular datasets like Pascal VOC [9], ILSVRC [8] and MS COCO [24]. However, this algorithm still performs well under human performances for the task of counting on datasets like MS COCO.

Current object detection methods use either region proposals [45] or sliding windows at multiple scales [10] to generate the initial set of candidate bounding boxes. 
An object classifier is run on these proposed boxes, the classification results are further refined by techniques like greedy non-maximal suppression and bounding box regression. Once the objects are detected accurately, counting them is trivial. However, in practice the post processing steps in object detection often require elaborate fine tuning and optimization. In contrast, in this article, we reformulate the problem of counting as a Bayesian estimation problem.

The simplest probabilistic models that describe the position of instances of a given object within an image are Poisson processes [19]. A (non uniform) Poisson process is fully characterized by a positive function over the image domain called an intensity. According to the definition of a Poisson process, the sum of the intensity over a sub-image provides the expected value as well as the variance of the number of instances within this sub-image. Specifically, the total number of instances of the object in the image can be estimated by summing the intensity over the whole image domain. In practice, the intensity is not available but can be estimated from data, thus providing an algorithmic solution to the counting problem.

One of the key issues, then, is the generation of the data necessary for a good estimation of the intensity function. How can this best be achieved? In this paper, we propose to leverage recent advances in object classification by running a CNN binary classifier for the object of interest over a dense pixel grid. Despite the overall good quality of the CNN output, visual inspection of the resulting images shows that there are multiple responses per instance, that these "blobs" of responses might be intersecting, of different sizes and corrupted by noise such that the problem of estimating the intensity from this data is not trivial. Note that a crude way of estimating the count directly from the CNN detection output would be to detect the connected components, or count the number of local maxima, but this involves ad-hoc smoothing and thresholding of the classifier output, the parameters of which can only be computed empirically; they also cannot be easily transferred from one object type to the other or from one dataset to another. Note that there exists many sophisticated methods ([30], [32]) with efficient global regularization that develop density estimates from saliency map. Our work aims to provide an alternate approach to estimating this density map from the CNN detection output using Cox modeling.

Since regularization is needed, we adopt a Bayesian point of view. A positive function, called the intensity is used to describe the expected number of instances of an object within a surface element of the image domain. We model the intensity as a random positive function.
A Gaussian Process (GP) [35] provides a random function, and a link function maps the GP into a probability distribution over intensities. This construction is standard, dating back to the Cox process, introduced by David R. Cox in 1955 [5]. However, the applications are recent due to the relatively high computational burden for computing the posterior and estimating the hyper parameters. These applications include reinsurance pricing, portfolio optimization [7], and estimating crime maps in Chicago [12]. The application to visual counting is new to the best of our knowledge.

Inference with Cox processes is complicated since there is no conjugated model. Instead, an approximate method must be used, the simplest of which is the Laplace method [27]. It consists in approximating the posterior distribution over the GP with a GP. The posterior mean is obtained by solving a convex minimization problem using the Newton method. Still, this method cannot be used directly because it scales poorly with the dimension of the image. The same problem occurs in GP classification [35] as well, where the computational and storage costs are $O\left(n^{3}\right)$ and $O\left(n^{2}\right)$ respectively. Saatçi [40] presents a technique for using Kronecker algebra which makes the GP inference more tractable, with $O\left(n^{3 / 2}\right)$ measurements and $O(n)$ memory requirements without any loss of accuracy. This method requires that the input locations remain on a lattice, but this is not a limitation for computer vision applications as this is a natural structure for images.

We make the following three contributions in this work: First, we re-formulate the problem of visual counting using Cox processes; second, we incorporate Kronecker algebra into this model for efficiency; and finally, we present a tractable algorithm for the computation of the posterior distribution that facilitates counting. The remainder of this paper is organized as follows: In section 2, we present a survey of the related work, which is followed by a description of our algorithm and methodology in section 3. Section 4 demonstrates the performance of the proposed algorithm on real and simulated data, and section 5 concludes the paper.

\section{Related Work in Visual Counting}

A large body of work in object counting falls into three categories, 1) counting by density estimation, 2) counting by regression and 3) counting by detection.

Counting by density estimation: In this class of solutions, the counting problem is reformulated as the task of estimating an image density, the integral of which provides the count of objects in the image. These methods [3], [18], [23], [11], [2] typically learn a mapping between local image features and object density, which al- 
lows the estimation of a density map for new unseen images. In [23], the density is a linear function of a feature vector associated with each pixel, which is estimated by minimizing a quadratic cost function. In contrast, the work in [11] uses a regression random forest that learns the mapping between image patch features and patch density. The image density is then obtained by averaging over patch-wise density predictions. Most of these methods use domain-specific visual features based on SIFT [26] and HOG [6] as these were proposed prior to the widespread use of CNN features. The accuracy of these methods depend significantly on the choice of image features. [2] proposes an interactive counting strategy where the framework learns from annotated regions of the image and computes a density map for the nonannotated regions in the same image, allowing the user to inspect the results and refine the estimations. These algorithms, having primarily been developed for crowd analysis, are generally untested outside this domain.

Counting by regression: Here, the object count is estimated by mapping from a set of global features to the integer counts, instead of estimating the count by integrating a density function. In [20] blob size histograms and edge orientations are used as features for estimating the number of pedestrians in an image. [28] and [4] use edge features and texture information based on grey-level transition probabilities in order to directly estimate crowd density using neural nets. These approaches typically discard information about the location of objects, using only the total count for learning.

Counting by detection: In this category of solutions, it is assumed that there is a visual object detector that is tuned to find individual instances of the object. Once the instances are localized, counting becomes a trivial task. Classification networks like Alexnet [21] and $V G$ GNet [42] have the ability to classify images, but are limited by the fact that they require a fixed-size input image, which means that the image sub-regions should be made to fit either via cropping or warping, leading to distortion. SPP-Net[17] solved this problem by using a convolutional feature map from the entire image and then pooling features in arbitrary sub-regions of the image to generate the fixed length representation required in the later layers. The more recent YOLO [36] [37] reframes detection as a regression problem, using a single convolutional network to predict a set of bounding boxes and class probabilities. Faster-RCNN [38] built up on their earlier work [15] [14] [17] for object detection with region proposal networks, and localize objects with a $m A P$ (mean average precision) of $42.7 \%$ on the MS COCO dataset. When applied to counting birds in the same dataset, Faster-RCNN is found to have a root mean square error (RMSE) of 2 (see Table 2). The dis- parity in this and the results obtained from the other object types suggests there is scope for further improvement.

In addition to the above categories, there have been many recent interesting work in counting using Bayesian modeling. Pham et al. [33] employs point process inference for large scale object detection and counting, while in [13], a Bayesian marked point process is developed to detect and count people in crowded scenes, leading to an estimate of the count, location and pose of each person in the scene. Point processes allow convenient modeling and analysis of spatial data, the object configuration and the interaction between objects. Marked point processes extend point processes by adding specific marks that associate a parametric object to each point. [22], [46] and [32] use models that allow the representation of images in terms of simple geometric features, with the goal of estimating counts.

CNN-based counting approaches offer powerful improvements over methods that rely on hand-crafted representations [44]. Wang et al. [48] developed an end-toend CNN regression model for counting people in images of extremely dense crowds. Walach and Wolf [47] learn a density map estimated directly from the input image employing layered boosting and selective sampling. Sindagi and Patel [43], on the other hand, use a cascaded network of CNNs to jointly learn crowd count classification and density map estimation in densely crowded scenes. [41] propose a switching CNN that leverages intra-image crowd density variation to improve crowd count estimates. Rubio and Sastre [31] developed a Counting CNN where the network learns to map the appearance of image patches to their object density maps.

The proposed algorithm combines the benefits of both counting detection and by density estimation approaches. We do not hand-craft the image features, but instead use the pre-trained CNN classifiers to provide our measurements. Lastly, since we compute a posterior density over the entire image, it is possible to estimate the count in image sub-regions by integrating the posterior over that region.

\section{Methods and Algorithms}

\subsection{Overview}

The block diagram in Figure 1 provides an overview of the process involved in estimating the number of instances of the object of interest in an input image. First, a set of bounding boxes is selected from the image, on which we run a classifier. The classifier is trained to classify each bounding box for the presence or absence 


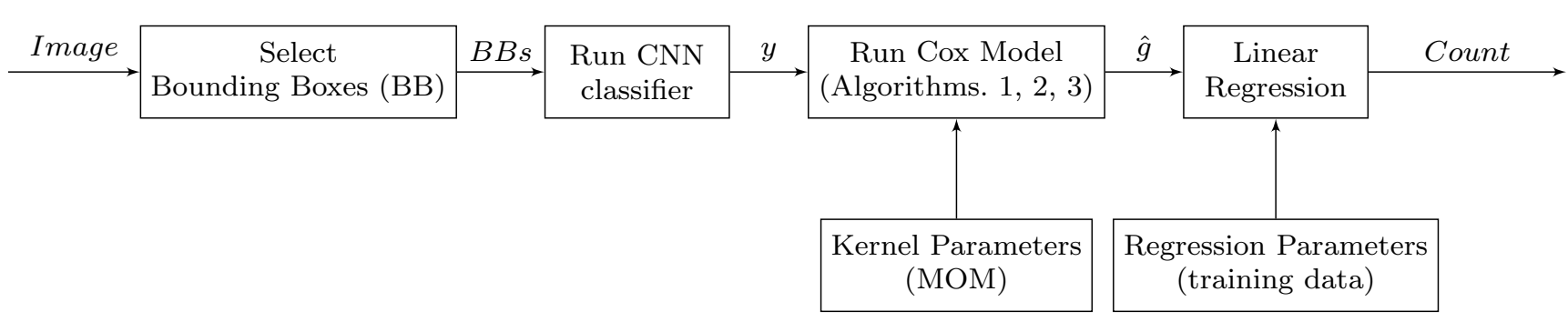

Fig. 1 Block diagram for the counting work-flow.

of the object type that we are interested in enumerating. The scaled classifier scores from this initial set of bounding boxes are the measurements $y$ used in the sections that follow. In our Cox modeling, these observations $y$ are functionally related to the intensity of the Poisson process. (The details of this formulation are described in sections 3.2 and 3.3, and the likelihood model is elaborated in section 3.4 ). The posterior distribution given the observations $y$ is expressed using Bayes formula in section 3.5, and the posterior mean $\hat{g}$ is computed using Algorithms 1, 2 and 3 (as detailed in sections 3.6 and 3.7). Finally, the integral of the posterior mean $\hat{g}$ is mapped using linear regression to find the final estimated count.

\subsection{A Brief Introduction to Cox Processes and Gaussian Processes}

Cox processes are also called Mixed Poisson Processes or Doubly Stochastic Poisson Processes. A stochastic process [39] $\mathcal{X}=\{X(t), t \in T\}$ is a collection of random variables defined on a common probability space $(\Omega, \mathcal{F}, \mathcal{P})$, where $\Omega$ is the sample space, $\mathcal{F}$ is a $\sigma$-algebra of subsets of $\Omega$, and $\mathcal{P}$ is a non-negative probability measure on $(\Omega, \mathcal{F})$ with total mass $\mathcal{P}(\Omega)=1$. For each $t$ in the set $T, X(t)$ is a random variable that represents the state of the process at index $t$, and $t$ is often interpreted as either time or space. The simplest of stochastic processes is a Bernoulli process, which is a sequence of independent and identically distributed random variables, each of which can take a value of zero or one based on probability $p$ and $1-p$ respectively.

A Gaussian Process (GP) [35], on the other hand, is defined as a collection of random variables, any finite number of which have a joint Gaussian distribution. A Gaussian Process can be written as, $g(x) \sim$ $G P\left(\bar{g}(x), k\left(x, x^{\prime}\right)\right)$, and is completely specified by its mean function $\bar{g}(x)$ and a positive definite kernel $k\left(x, x^{\prime}\right)$. We say that $g \sim G P(\bar{g}, k)$ is a GP when for any collection of points on the input space $\left(x_{1}, \ldots, x_{n}\right)$, the vector of real numbers $\left(g\left(x_{1}\right), \ldots, g\left(x_{n}\right)\right)$ is distributed as a multivariate Gaussian with mean $\bar{g}$ and covariance
$\Sigma$, such that $\Sigma_{i j}=k\left(x_{i}, x_{j}\right)$. Recall that a positive definite kernel is a function of two arguments such that the matrix $\Sigma$ is a covariance matrix. The marginalization property of the GP follows directly from this specification of the covariance matrix. This property means that if the GP specifies $\left(g\left(x_{1}\right), g\left(x_{2}\right)\right) \sim \mathcal{N}(\bar{g}, \Sigma)$, then it must also specify $g\left(x_{1}\right) \sim \mathcal{N}\left(\overline{g_{1}}, \Sigma_{11}\right)$, where $\Sigma_{11}$ is the relevant sub-matrix of $\Sigma$. Following the notation in Rasmussen and Williams [35], given a dataset $D$ of $n$ observations, $D=\left\{\left(\mathbf{x}_{i}, y_{i}\right) \mid i=1, \ldots, n\right\}=$ $(X, \mathbf{y})$, we assume that the relationship between the input data $X$ and the target $\mathbf{y}$ is governed by a latent GP function $g(x) \sim G P(\bar{g}, k)$, and $p(y(x) \mid g(x))$ is the observation model or the likelihood. In Gaussian process regression, the goal is to find a predictive distribution $p\left(g_{*} \mid y, X, X_{*}\right)$ for any new test input set $X_{*}$. In the standard GP regression formulation, the observation model is Gaussian, which when combined with a Gaussian prior gives rise to a Gaussian posterior, and the posterior distribution remains analytically tractable. However, for most other applications of Gaussian Processes, including GP classification, the solution is more demanding since the likelihood is typically nonGaussian.

Poisson Processes are stochastic processes for collections of points on a domain, the number of points in this collection being also random. A Poisson Process is characterized by a rate or intensity function $\lambda$. If $\lambda$ is constant over the domain $\Omega$, the process is said to be stationary or homogeneous, and if $\lambda(t)$ varies with time or space, the process is inhomogeneous. In a doubly stochastic process, the observed random variables are modeled in two steps: in the first step, the random variables are defined using a stochastic process characterized by one or more parameters, and in the second step, the parameters themselves are treated as random variables. A Cox process, also known as a doubly stochastic Poisson Process is a stochastic process which is a generalization of a Poisson Process where the time(or space)-dependent intensity $\lambda(t)$ is itself a stochastic process. In the case of a Gaussian Cox Process, this intensity is obtained by mapping a Gaussian Process to a positive function using a link function. Ex- 
amples of link functions include the square, the exponential, the sigmoid, and the logit function. Conditional on the intensity $\lambda$, the number of instances within the region $\Omega$ is,

$N(\Omega) \mid \lambda=$ Poisson $\left(\int_{\Omega} \lambda(s) d s\right)$

where Poisson $(u)$ stands for the Poisson distribution with parameter $u$.

\subsection{Problem Formulation}

Let $\theta$ be a doubly stochastic Poisson Process (PP) with intensity $\lambda: \theta \sim P P(\lambda)$ over the domain $\Omega=[0,1]^{d}$. In our experiments, $d=2$. The random intensity function $\lambda$ is obtained by mapping a Gaussian Process (GP) defined over $\Omega$ to a positive function. Let $g \sim G P(\bar{g}, \Sigma)$, where $\bar{g}$ is a function $\Omega \mapsto \mathbb{R}$, and $\Sigma$ is a positive definite kernel over $\Omega \times \Omega$. We define $\lambda()=.\alpha \phi(g()$.$) , where$ $\phi$ is a function $\mathbb{R} \mapsto \mathbb{R}^{+}$. Examples include $\phi(g)=e^{g}$ and $\phi(g)=g^{2} . \alpha>0$ is a scaling factor that together with $\bar{g}$ and $\Sigma$ control the expected number of counts in the domain.

Consider now a finite grid $\mathcal{D}$ defined over the continuous domain $\Omega$. Moreover, assume that $\mathcal{D}=\mathcal{D}_{1} \times$ $\ldots \times \mathcal{D}_{d}$. That is, $\mathcal{D}$ is the cross product of $d$ onedimensional grids. This is key to ensuring the scalability of the model. An example for $\mathcal{D}$ when $d=2$ entails choosing the centers of the pixels of a digital image.

This grid is chosen such that measurements are taken for each of the points in $\mathcal{D}$, or a subset thereof. Notate $n$, the size of $\mathcal{D}$, and $\tilde{n}$, with $\tilde{n} \leq n$, the number of observed measurements. The locations of the measurements are $x=\left(x_{1}, \ldots, x_{\tilde{n}}\right)$, where $x_{m} \in \mathcal{D}$. The measurements themselves are notated $y=\left(y_{1}, \ldots, y_{\tilde{n}}\right)$. There is no restriction on measurement type: these could be counts, or real or vector valued measurements.

The posterior on $g$ given the observations $y$ is, using the Bayes formula,

$$
\ln p(g \mid y) \propto \ln p(g)+\ln p(y \mid g)
$$

\subsection{The Forward Model}

In order to define a probabilistic model for $y$ given $g$, let us define $g_{m}=g\left(x_{m}\right)$ and $\lambda_{m}=\alpha \phi\left(g_{m}\right), 1 \leq m \leq \tilde{n}$. In the standard Cox process, the observation $y_{m}$ at $x_{m}$ is a sample from a Poisson distribution with mean $\lambda_{m}$, for $1 \leq m \leq \tilde{n}$. In visual counting however, $y_{m}$ is a number in the range $[0,1]$. It is the response of a classifier. Moreover, a single instance of an object typically generates a large number of positive responses in a neighborhood of this instance (examples are provided in Figure 5). The number of positive responses depends on the size, in pixel of these instances. A detailed modeling of this process would require a hierarchical model and several parameters. Instead, we opt for a simplified model in which a high probability corresponds to the situation where the observed value $y_{m}$ is close to the intensity $\lambda_{m}$. We also want this likelihood function $\ln p(y \mid g)$ to be concave so that its summation with the prior logarithm $\ln p(g)$ results in a concave function with a unique maximum. Eq. (3) below provides one such function,

$\ln p\left(y_{m} \mid g_{m}\right) \propto-\beta\left(g_{m}-\sqrt{y_{m}}\right)^{2 p}$,

for some $p \in\{1,2, \ldots\}$, and $\beta>0$ Note that the case $p=1$ corresponds to a Normal distribution. The case $p=2$ is also interesting. In this case, $p\left(y_{m} \mid g_{m}\right)$ has a "plateau" centered at $\sqrt{y_{m}}$. The size of the plateau depends on $\beta$. This is the model that we use in our experiments.

We now assume that for each $1 \leq m \leq \tilde{n}$,

$$
\begin{aligned}
\ln p(y \mid g) & =\sum_{m=1}^{\tilde{n}} \ln p\left(y_{m} \mid g\right) \\
& =\sum_{m=1}^{\tilde{n}} \ln p\left(y_{m} \mid g_{m}\right)
\end{aligned}
$$

In other words, the observations are conditionally independent given the intensity, and the observation $y_{m}$ depends on $\lambda$ only through $\lambda_{m}$, the intensity at $x_{m}$. We also assume that $\ln p\left(y_{m} \mid g_{m}\right)$ is a concave function of $g_{m}$. This is the case for the traditional Cox process, that is when $y_{m}$ is Poisson distributed with intensity $\lambda_{m}$ and $\phi(g)=g^{2}$. This is also the case for the model presented in Eq. (3).

\subsection{The Posterior Distribution}

The posterior on $g$ given the observations $y$ is,

$$
\ln p(g \mid y) \propto \ln p(g)+\ln p(y \mid g)
$$

Since in general $p(y \mid g)$ is non-Gaussian, $p(g \mid y)$ is non-Gaussian, and thus cannot be computed analytically. Instead, following [12] and [35], we use a sophisticated numerical method which provides a tractable approximation of the distribution of $p(g \mid y)$. Specifically, we use the Laplace method together with Kronecker algebra and pre-conditioning to compute a Gaussian approximation of $p(g \mid y)$. The posterior mean of $g$ given $y$ is notated $\hat{g}$ and the $(n, n)$ posterior covariance matrix of $g$ is notated $A$. 
Let $\Phi(g)=\ln p(g \mid y)$; in order to find the posterior mean $\hat{g}$ that maximizes the log posterior $\Phi(g)$, Laplace approximation uses the second order Taylor series expansion of $\Phi(g)$ about the point $g=\hat{g}$,

$\Phi(g) \simeq \Phi(\hat{g})+\frac{1}{2}(g-\hat{g})^{T} \nabla \nabla \Phi(\hat{g})(g-\hat{g})$

Note that the first order term in Eq. (5) is zero since the gradient, $\nabla \Phi(\hat{g})=0$ at the maximum. Noting that Eq. (5) is log-Gaussian, we get,

$p(g \mid y) \approx \mathcal{N}\left(\hat{g},-(\nabla \nabla \Phi(\hat{g}))^{-1}\right) \quad=\mathcal{N}(\hat{g}, A)$

Differentiating $\Phi(g)$ w.r.t. $g$ provides

$$
\begin{aligned}
\nabla \Phi(g) & =-\Sigma^{-1}(g-\bar{g})+\nabla_{g} \ln p(y \mid g) \\
\nabla \nabla \Phi(g) & =-\Sigma^{-1}+\nabla \nabla_{g} \ln p(y \mid g) \\
& =-\Sigma^{-1}-W
\end{aligned}
$$

where $W=-\nabla \nabla_{g} \ln p(y \mid g)$. The posterior covariance is $A=\left(\Sigma^{-1}+W\right)^{-1}$. Note that since $\ln p\left(y_{m} \mid g_{m}\right)$ is a concave function of $g_{m}, W$ is semi-definite positive and $A$ is well defined. (See Section. 3.4 and Appendix 6.2)

In the Laplace method, $\hat{g}$ is computed using Newton's algorithm. Following [35], the Newton iteration for the Laplace Approximation can be computed in the following manner which improves the numerical stability of the algorithm:

$$
\begin{aligned}
g^{(t+1)} & =g^{(t)}-\left(\nabla \nabla \Phi\left(g^{(t)}\right)\right)^{-1} \nabla \Phi\left(g^{(t)}\right) \\
& =\left(\Sigma^{-1}+W\right)^{-1}\left[W g^{(t)}+\right. \\
& \left.\Sigma^{-1} \bar{g}+\nabla_{g} \ln p\left(y \mid g^{(t)}\right)\right] \\
& =\Sigma\left(I-W^{1 / 2} B^{-1} W^{1 / 2} \Sigma\right)\left[W g^{(t)}+\right. \\
& \left.\Sigma^{-1} \bar{g}+\nabla_{g} \ln p\left(y \mid g^{(t)}\right)\right] \\
& =\Sigma\left[b-W^{1 / 2} B^{-1} W^{1 / 2} \Sigma b\right] \\
& =\Sigma a
\end{aligned}
$$

where, $B=I+W^{1 / 2} \Sigma W^{1 / 2}, a=b-W^{1 / 2} B^{-1} W^{1 / 2} \Sigma b$, $b=W g^{(t)}+\Sigma^{-1} \bar{g}+\nabla_{g} \ln p\left(y \mid g^{(t)}\right)$.

Note that $W$ is a non-negative diagonal matrix and $B$ is symmetric positive definite.

3.6 Tractability: Kronecker Algebra and Separable Kernels

Solving Eq. (8) directly is not practical since it requires manipulation of matrices of size $(n, n)$. To tackle this, we make use of covariance functions that can be decomposed as a product of separable functions over dimensions $i=1, \ldots, d$ as,

$k\left(x, x^{\prime}\right)=\prod_{i=1}^{d} k_{i}\left(x^{(i)}, x^{(i)}\right)$

where $x^{(i)}$ is the $i^{\text {th }}$ dimensional element of input $x$. Such kernels are called tensor product kernels. Note that it is a requirement of the method (see Algorithm 2 and 3) that the covariance kernel be decomposable as a product. While this is not true of all tensor product kernels, the number of compliant kernels is sufficiently large that this does not present a serious limitation. Examples include the exponential kernel, squared exponential kernel and the Matérn kernel (See [35] and [40]). The squared exponential kernel can be decomposed as,

$$
\begin{aligned}
k\left(x, x^{\prime}\right) & =\sigma^{2} \exp \left(-\frac{\left\|x-x^{\prime}\right\|^{2}}{2 l^{2}}\right) \\
& =\sigma^{2} \exp \left(-\sum_{i=1}^{d} \frac{\left(x^{(i)}-x^{\prime(i)}\right)^{2}}{2 l^{2}}\right) \\
& =\prod_{i=1}^{d} \sigma^{2 / d} \exp \left(-\frac{\left(x^{(i)}-x^{\prime(i)}\right)^{2}}{2 l^{2}}\right)
\end{aligned}
$$

The tensor product covariance function evaluated over a grid can be written as a Kronecker product of $d$ smaller covariance matrices:

$$
\Sigma=\bigotimes_{i=1}^{d} \Sigma_{i}
$$

where $\Sigma_{i}$ is the covariance matrix on each dimension and represents the correlation between any two observations along that dimension. The $d$ matrices are of sizes $\left(n_{1}, n_{1}\right) \ldots\left(n_{d}, n_{d}\right)$, where $n_{i}$ is the size of $\mathcal{D}_{i}$ and $n=n_{1} \times \ldots \times n_{d}$. Plugging Eq. (11) into Eq. (8), the Newton Iteration becomes

$$
g^{(t+1)}=\bigotimes_{i=1}^{d} \Sigma_{i} a
$$

Computing Eq. (12) efficiently requires the use the following property of Kronecker algebra:

$$
\operatorname{vec}\left(A X B^{T}\right)=(B \bigotimes A) \operatorname{vec}(X)
$$

where the operator $\operatorname{vec}(X)$ is a column-wise stacking of the square matrix $X$. We use the algorithm from Saatçi [40] to compute this product, which is reproduced below for completeness (Algorithm 1). 


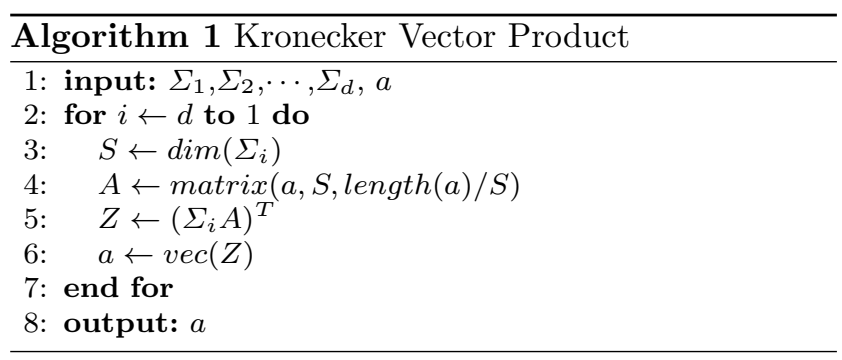

Note $\Sigma_{i}$ is a $n_{i} \times n_{i}$ matrix and $a$ is a $n \times 1$ vector. Line 3 assigns $n_{i}$ to $S$; Line 4 rewrites the vector $a$ as a matrix with $S$ rows and length $(a) / S$ columns.

\subsection{Computation of the Posterior}

We now incorporate the ideas presented in Section. 3.5 into the Newton's method, for computational and storage efficiency. In Newton iteration we need to evaluate $B^{-1}$. The Cholesky decomposition would require $\mathcal{O}\left(n^{3}\right)$ time and $\mathcal{O}\left(n^{2}\right)$ storage. However, due to the Kronecker structure of the covariance matrix $\Sigma$, the Conjugate Gradient Method only requires $\mathcal{O}\left(d n^{\frac{d+1}{d}}\right)$ time and $\mathcal{O}\left(d n^{\frac{2}{d}}\right)$ storage, which is also employed in Flaxman et al. [12]. We now summarize the Conjugate Gradient Algorithm 2 adapted for the Kronecker structure.

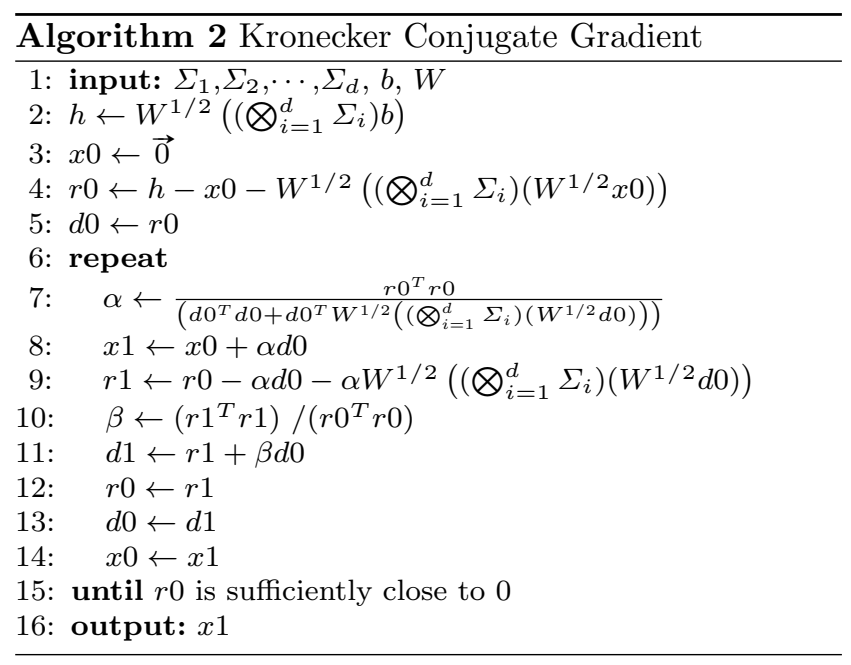

Based on Algorithms 1 and 2, the Newton Iteration in Eq. (8) can be implemented by the Algorithm 3. Computing $a=\left[b-W^{1 / 2} B^{-1} W^{1 / 2} \Sigma b\right]$ in Eq. (8) involves finding $B^{-1}$ which is computationally expensive. To overcome this, we set $B x=W^{1 / 2} \Sigma b$, and solve for $x$ using Algorithm 2, as shown below in line 6 of Algorithm 3 .

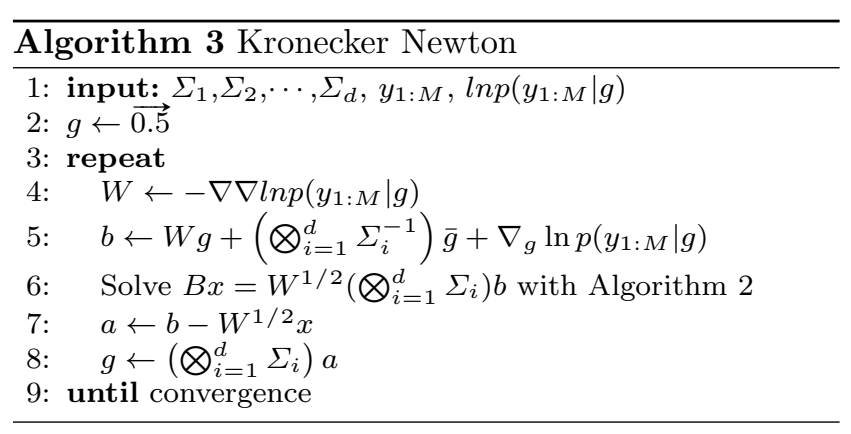

3.8 Method of Moments for Kernel Parameter Estimation

The traditional method for estimating the parameters of the kernel involves using the method of maximum likelihood, see [35]. However, this method is computationally expensive and requires a sophisticated numerical implementation. Instead, in the case of the squared exponential kernel, we show that the Method of Moments (MoM) provides simple analytical expressions.

We notate the squared exponential kernel,

$k_{S E}\left(x, x^{\prime}\right)=\sigma^{2} \exp \left(-\frac{\left\|x-x^{\prime}\right\|^{2}}{2 l^{2}}\right)$

We assume that we observe $m$ independent samples from the prior distribution, each over a domain of size $n$. Let $N_{i}$ is the number of instances for sample $i$, for $1 \leq i \leq m$. Let $\bar{N}$ and $s_{N}$ be respectively the sample mean and the sample standard deviation of $N_{1}, \ldots, N_{m}$. $\sigma$ and $l$ are then estimated with

$\hat{\sigma}^{2}=\frac{\bar{N}}{n}$ and $\hat{l}^{2}=\frac{n\left(s_{N}^{2}-\bar{N}\right)}{2 \pi \bar{N}^{2}}$

The derivation uses properties of the Poisson process and is presented in Appendix 6.1. Simulation results using MoM are provided in Section. 4.1 and Table. 1. The same technique is used to find the parameters of the kernel for the experiments on real image data, as illustrated in Section. 4.2.

\section{Experiments and Results}

\subsection{Simulation}

In this section, we demonstrate our algorithm on simulated image data. First of all, we verify the method of moments(MoM) described in section 3.8 for the estimation of the parameters of the squared exponential kernel. We set a range of values for $\sigma$ and $l$ of the kernel, and sample object instances using Cox processes in 1000 images according to this prior. Specifically, we 
Table 1 MoM for prior parameters $\sigma$ and $l$

\begin{tabular}{cccc}
\hline$l=1.5$ & $\hat{\sigma} \operatorname{mean}(\mathrm{std})$ & $\sigma=0.1$ & $\hat{l}$ mean(std) \\
\hline$\sigma=0.1$ & $0.0999(0.0005)$ & $l=0.5$ & $0.8142(0.3386)$ \\
$\sigma=0.5$ & $0.5002(0.0011)$ & $l=1.0$ & $0.9730(0.3554)$ \\
$\sigma=1.0$ & $1.0002(0.0023)$ & $l=1.5$ & $1.4138(0.2966)$ \\
$\sigma=1.5$ & $1.4997(0.0030)$ & $l=2.0$ & $1.9240(0.1960)$ \\
$\sigma=2.0$ & $1.9995(0.0038)$ & $l=3.0$ & $2.8105(0.1925)$ \\
\hline
\end{tabular}

draw 1000 samples $g$ from multivariate normal distributions characterized by the kernel, calculate the intensity $\lambda$ from $g$ using the link function $\phi(g)=g^{2}$, and then sample the number of instances $N_{i}$ from a Poisson distribution. We repeat this process 100 times, estimating $\hat{l}$ and $\hat{\sigma}$ in every iteration of 1000 images each. Table 1 shows the mean and standard deviation of $\hat{l}$ and $\hat{\sigma}$. In the first column of the table, we fix the value for $l=1.5$ and use different values for $\sigma$. The second column shows the mean and standard deviation of the estimates of $\sigma$ corresponding to each $\sigma$ value in the first column. Column 3 and 4 show similar results for the parameter $l$. These results indicate that the method of moments provide good estimates for the kernel parameters.

In order to simulate the computation of the posterior intensity and the subsequent estimation of the number of object instances, we generate images of size $(30 \times 30)$ over which instances are assigned using the following scheme:

- Sample according to a Poisson process over the image domain with constant intensity $\lambda=5 /(30 \times 30)$ such that in average, 5 instances per image are sampled.

- Set a bounding box centered at each object instance with a random width and height ranging from 3 to 10.

- In real-world images, the positive responses generated by the CNN classifier are clustered in blob-like structures as shown in the second column of Figure 5 . To simulate this behavior, we generate object instances over the bounding box using a Bernoulli distribution with probability $p$. Specifically, since each instance generates multiple measurements, and in order that the total intensity be close to the expected number of instances, we set each measurement $y_{m}$ in the bounding box to $y_{m}=1 /(p * N)$ with probability $p=0.8$ and $y_{m}=0$ with probability $1-p=0.2$ (where $N$ is the total number of measurements in the bounding box.)

We use the squared exponential kernel, $k_{S E}\left(x, x^{\prime}\right)=$ $\sigma^{2} \exp \left(-\frac{\left\|x-x^{\prime}\right\|^{2}}{2 l^{2}}\right)$ with parameters $\sigma=2.4$ and $l=$ 2 as the GP covariance function for this simulation. The top image of Figure 2 shows a function drawn at random from the GP prior, and the image below is the corresponding prior of the intensity.
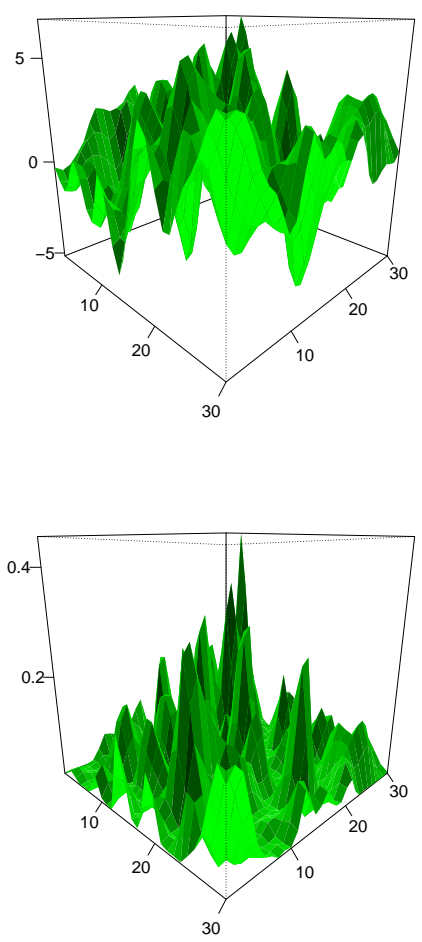

Fig. 2 (top) The prior function $g$ drawn at random from a GP with a squared exponential covariance function; the input here is two dimensional, and (bottom) the corresponding positive prior intensity, $\lambda=\alpha g^{2}$

Figure 3 shows results of one of the simulations. The red triangles in each bounding box stands for the detected instances of the object. Since the outline of the object instance is usually not exactly a box, there are some missed measurements in each bounding box generated randomly by a Bernoulli distribution. The middle image in Figure 3 shows the posterior intensity, the black pixels indicate that the corresponding intensity of those locations are close to 0 . The rightmost image of Figure 3 displays the posterior intensity in a 3D graph. Note that Figure 3 demonstrates the situation where some of the object instances partially overlap and this overlapping is also reflected clearly in the posterior intensity in the same figure.

We replicate this procedure two thousand times and compute the total posterior intensity for each iteration. A standard linear regression equation is then used to model the relation between the total intensity and the true number of instances. Note that a more sophisticated forward model than the one described in section 

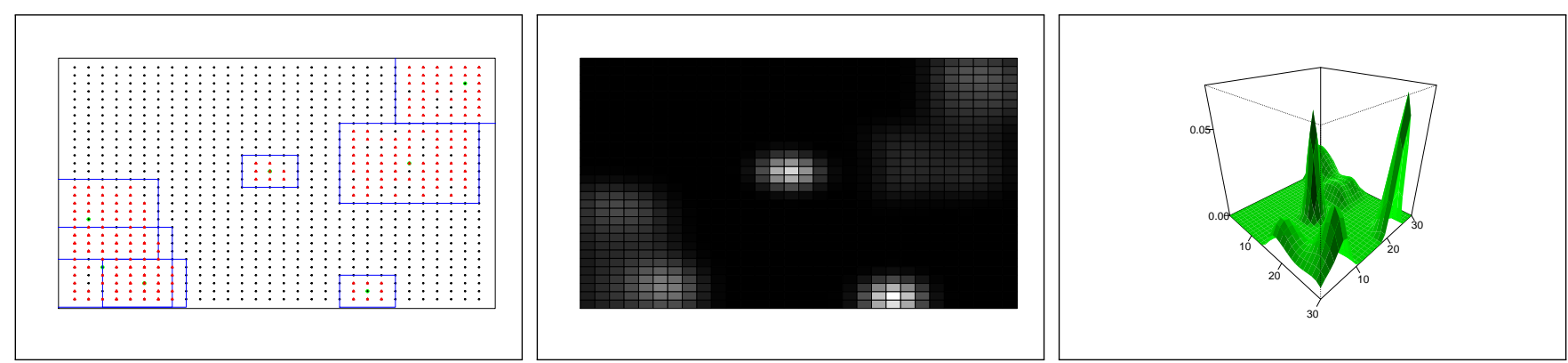

Fig. 3 Simulation results: (left) A simulated input image with 8 object instances, some of them overlapping. The blue rectangles show the bounding box annotations around the object. The red triangles in each bounding box stand for the detected instances of the object; this simulates the classifier results as there are typically multiple hits per instance. (middle) The posterior intensity computed by our algorithm and (right) The same posterior intensity as a 3D plot.

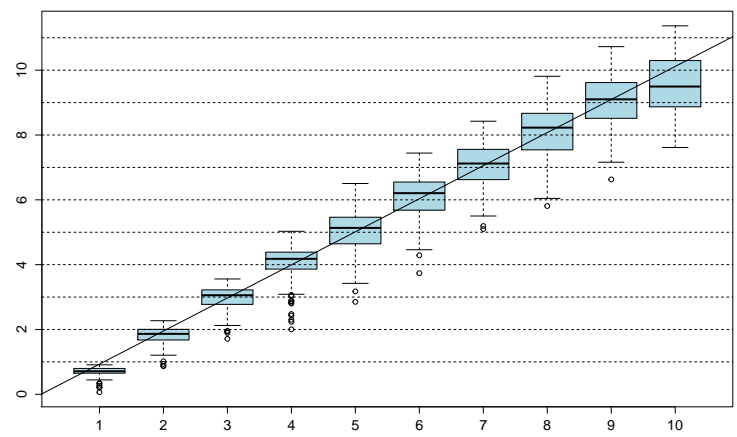

Fig. 4 Simulation Count result: The true count(x-axis) is plotted against the count estimated(y-axis) by the proposed algorithm over 2000 simulated images. (The number of object instances per image varies from 1 to 10 in this simulation.)

3.4 would potentially allow to compute the number of instances simply by integrating the estimated intensity. However, this would likely be obtained at some non negligible computation cost. According to the regression equation, we estimate the number of objects in each simulated image. The results are presented in Figure 4, the horizontal axis indicates the actual number of instances and the vertical axis is the estimated count. This box plot shows that the median of the estimated count is very close to the actual number of instances.

\subsection{Application to Real Images}

We now present the results of our algorithm on images from the MS COCO [24] dataset. In general, our approach works for all categories of objects, provided we have a classifier for that particular class. In this section, we demonstrate our results on three classes of objects from MS COCO dataset: "bird", "motorcycle" and "sheep". These classes were specifically chosen due to their datasets containing a satisfactory number of images with multiple instances of the same object type. In contrast, the "cat" or "table" datasets are predominantly comprised of solitary-instance images, and hence are not particularly challenging for our algorithm. (See the first column of Figure 5 for example images from COCO dataset.)

We use the squared exponential kernel as the covariance function for the GP prior, estimating the parameters $\sigma$ and $l$ using Eq. (15) derived in section 3.8. The sample mean and standard deviation used in this equation are computed from data using the ground truth bounding boxes annotations from COCO dataset. This is done for each class separately. We use a $100 \times 100$ grid over the image for all of our experiments with MS COCO. Specifically the grid dimension, $d=2$, size of the grid, $n=n_{1} \times n_{2}=10000$, and $\Sigma_{i}$ is a matrix of size $100 \times 100$. Note that the full covariance matrix $\Sigma$ would be of size $10000 \times 10000$, but we neither store nor manipulate this large $\Sigma$ directly, as explained in section 3 .

We begin the classification process by dividing the input image of size $M \times N$ into a $\frac{M}{\Delta t_{h}} \times \frac{N}{\Delta t_{w}}=100 \times 100$ grid, where $\Delta t_{w}$ and $\Delta t_{h}$ are the pixel distances between grid centers along the width and height of the image respectively. Each point in the grid is the center of the observation bounding box. We then observe each grid center $m$ at 5 different bounding box sizes by running the classifier trained specifically for the object category of interest over the set of bounding boxes. If none of the bounding boxes contain the object instance, we set the corresponding answer $y_{m}$ to 0 . In real-world images, the positive responses generated by the CNN classifier are clustered in blob-like structures as shown in the second column of Figure 5. Since each instance generates multiple measurements, and in order that the total intensity be close to the expected number of instances, we scale the classification score to get the 

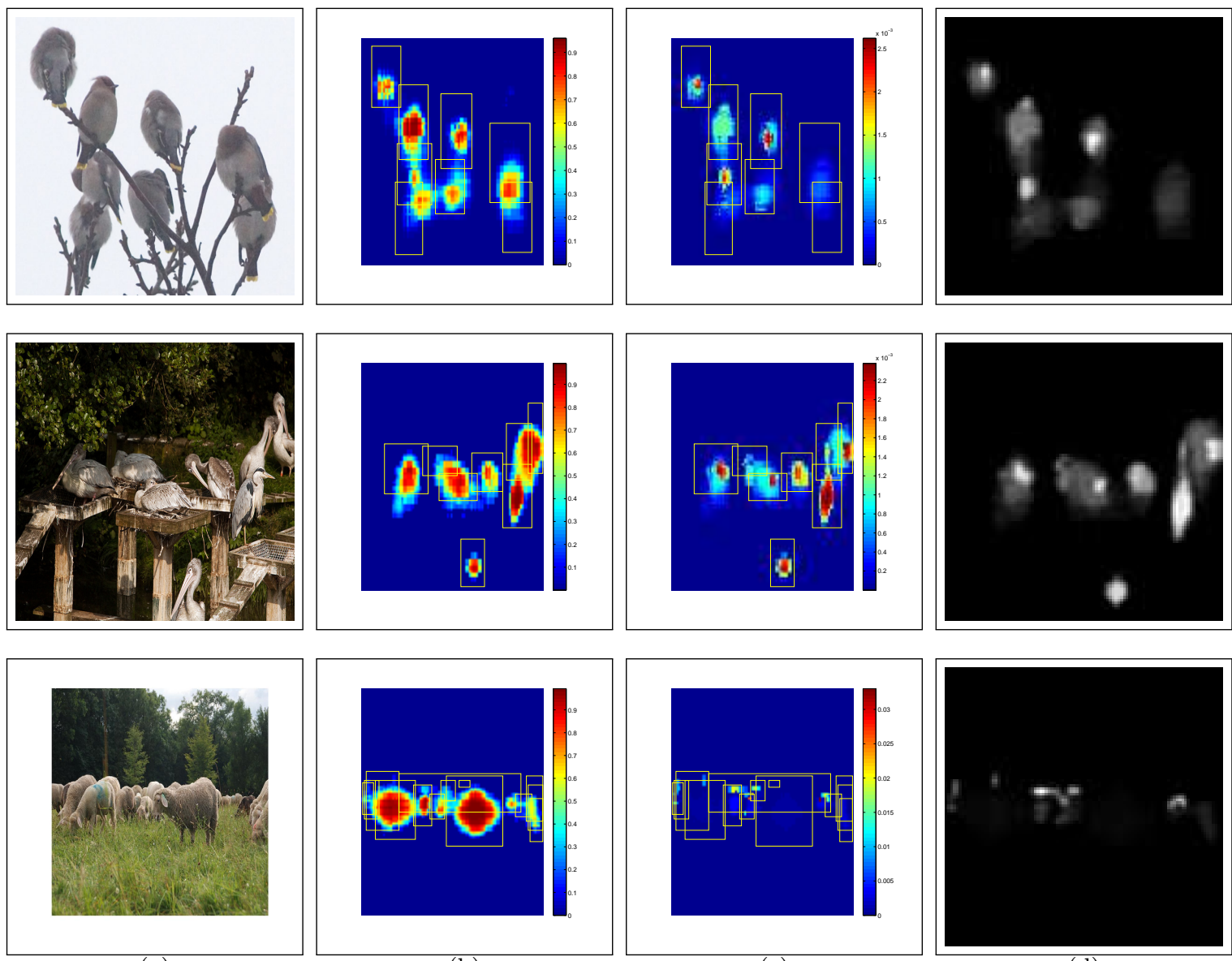

(b)
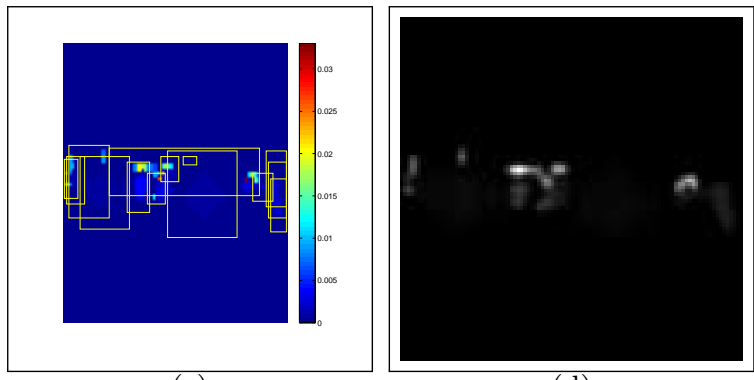

(d)

Fig. 5 Computation of the posterior intensity: (a) 3 sample images from the MS COCO dataset belonging to "bird" and "sheep" class; (b) Classification results: the classification scores after running the CNN classifier on a $100 \times 100$ grid, red and blue values indicating high and low confidence respectively; the ground truth bounding boxes annotations are shown in yellow, (c) the scaled classification results $y$ and (d) their corresponding posterior mean intensity computed by our algorithm.

observations $y_{m}$. If there is an instance detected at grid location $m$, we set $y_{m}=\frac{\Delta t_{h} \times \Delta t_{w} \times s}{M_{m} \times N_{m} \times p}$, where $\Delta t_{h}=\frac{M}{100}$ and $\Delta t_{w}=\frac{N}{100}$ are the grid distances, $s$ is the classification score, $M_{m} \times N_{m}$ is the size of the bounding box with the maximum classifier score and $p$ is the percentage of boxes in the neighborhood for which the classifier returns a true detection.

After we collect all the answers $y$, we compute the posterior distribution using Algorithms 1, 2, and 3 from section 3 . The average run time of our posterior computation algorithm implemented in MATLAB is 0.04 seconds on a CPU-only Intel Xeon desktop with 8 GB RAM. This was measured on MS COCO dataset that has a typical image size of $500 \times 500$ pixels.

Figure 5 shows 2 sample images from the test dataset, their ground truth bounding boxes in yellow, the answers $y$ from the classifier, and the posterior intensities computed by our algorithm. In order to estimate the final count, we calculate the integral of the posterior in- tensity over the space of bounding boxes, which is then fitted using linear regression. The results are shown in Figure 6 and Table 2. The box plots in Figure 6 show the true counts plotted against the estimated counts computed by both Cox and Faster RCNN on the MS COCO bird, sheep and motorcycle datasets. Our algorithm is robust to overlap, crowding and occlusion, the typical scenarios in which counting by detection fails. The last row of Figure 5 shows a difficult overlapping case for class type: "sheep". As is evident from the last column of Figure 5, our method can perform soft localization as well if we post-process the posterior intensity, for instance, by performing a non-maximal suppression over the set of bounding boxes. We intend to explore this framework for full localization in future work.

Table 2 details the root mean square error(RMSE) between the true number of objects and the count estimated by Cox, Faster-RCNN and Faster-RCNN with Regression. For the last baseline, we perform a linear 

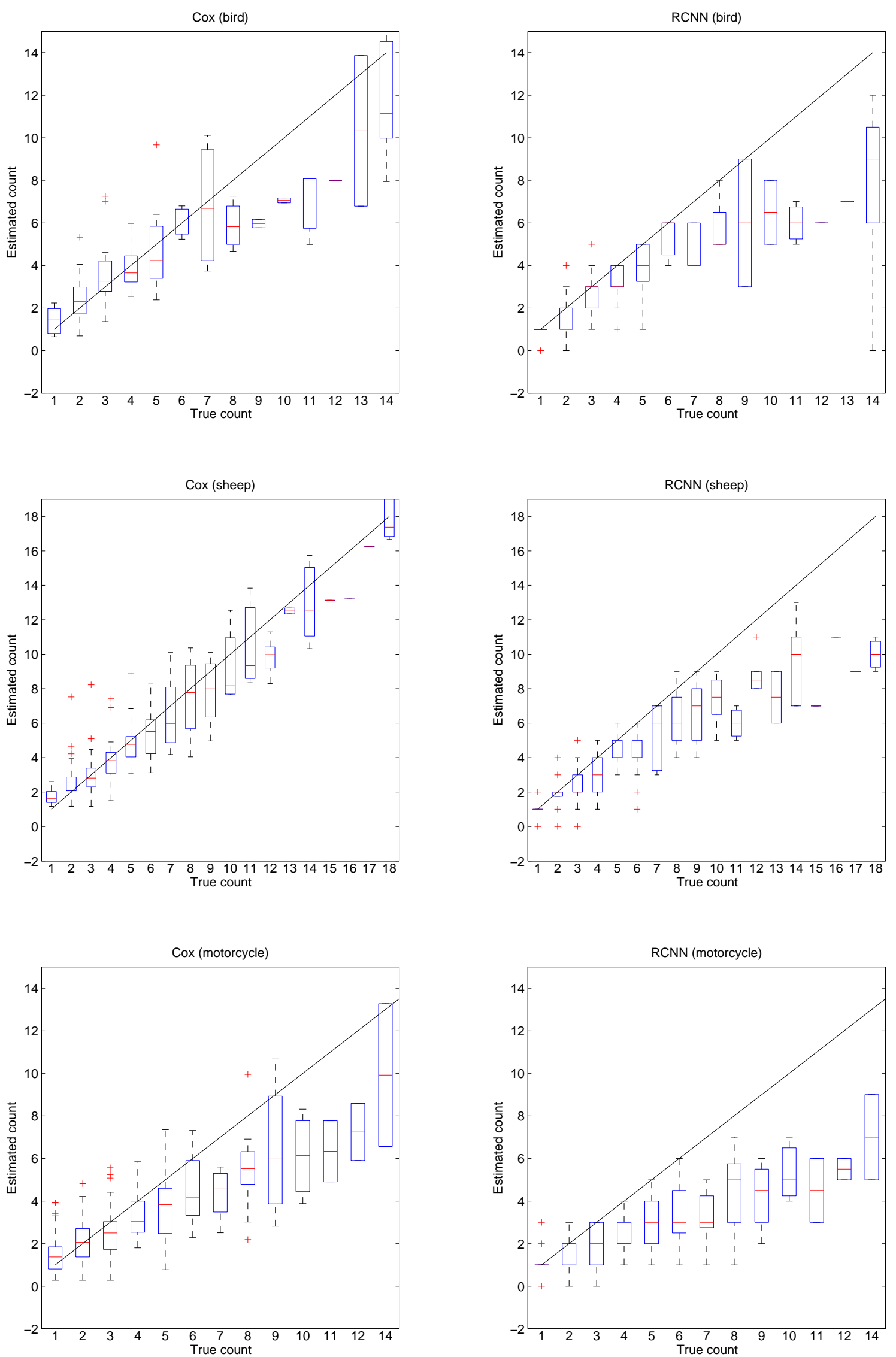

Fig. 6 Box plots that compare the counts estimated by Cox and Faster RCNN on images from MS COCO dataset. The number of instances per image ranges from 1 to 14 for both "bird" and "motorcycle" class, while it goes up to 18 for "sheep". On each box, the central red horizontal mark indicates the median, and the bottom and top edges of the box indicate the 25 th

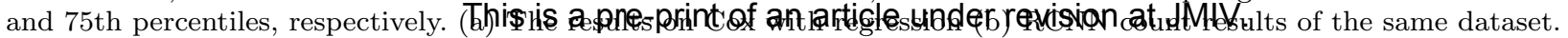


regression on the final results of Faster-RCNN. These numbers are shown in the same table, in the "Faster $R C N N$ Reg" row. For each category, we compute the RMSE on bootstrap samples and report the mean and standard deviation over all bootstrap iterations. The number of object instances per image, $k=[1,14]$; for each bootstrap sample we also compute RMSE separately for each $k$, find the mean per sample and finally calculate the mean and variance over all iterations. The result of these computations is shown in the last 3 rows of Table 2. The lower error rate observed for Cox over Faster RCNN in all cases suggests that our proposed counting algorithm is promising.

Table 2 RMSE mean (std) for counting. (The lowest error in each category shown in bold font.)

\begin{tabular}{lccc}
\hline Method & Bird & Motorbike & Sheep \\
\hline Cox & $\mathbf{1 . 8 5}(0.07)$ & $\mathbf{1 . 1 3}(0.14)$ & $\mathbf{1 . 4 4}(0.07)$ \\
Faster RCNN & $2.00(0.25)$ & $1.37(0.20)$ & $2.06(0.16)$ \\
Faster RCNN Reg & $2.01(0.17)$ & $1.15(0.14)$ & $1.62(0.11)$ \\
\hline Cox k & $\mathbf{2 . 3 3}(0.12)$ & $\mathbf{1 . 8 6}(0.34)$ & $\mathbf{1 . 8 4}(0.12)$ \\
Faster RCNN k & $2.77(0.29)$ & $2.79(0.42)$ & $3.03(0.32)$ \\
Faster RCNN Reg k & $2.53(0.13)$ & $1.99(0.38)$ & $2.04(0.22)$ \\
\hline
\end{tabular}

\section{Conclusion and Future Work}

We have presented a framework for estimating the number of instances of an object in an image, based on Cox processes. Evaluation using both synthetic and real data demonstrates empirically that our method scales well and improves upon the state-of-the-art.

In addition to counting, our algorithm allows for soft localization of objects. Since the location information is retained in the posterior intensity, our approach can be extended to precise object localization in the form of tight bounding boxes around the object. In addition, this work can be used as the basis for performing $o b$ ject localization by counting, building upon the ideas presented in [34] and [16]. These areas will be explored further in the next phase of our research.

\section{Acknowledgments}

This work was made possible in part thanks to Portland Institute for Computational Science and its resources acquired using NSF Grant DMS 1624776 and ARO Grant W911NF-16-1-0307, and the Department of Computer Science, Johns Hopkins University.

\section{Appendix}

6.1 Method of Moments for Kernel Parameter Estimation

Suppose the image domain is $\Omega$ with size $|\Omega|=a \times b$, where $a$ and $b$ are respectively the number of rows and columns of pixels in the image. Let $N(\Omega)$ be the number of instances of the object within $\Omega$. According to the model, $N(\Omega) \mid \lambda \sim$ Poisson $\left(\int_{\Omega} \lambda(s) d s\right)$, where $\lambda(s)=$ $\alpha g^{2}(s)$ and $s$ is a Gaussian Process $g \sim G P(0, K)$ with,

$$
K\left(s_{1}, s_{2}\right)=\sigma^{2} \exp \left\{-\frac{1}{2 l^{2}}\left\|s_{1}-s_{2}\right\|^{2}\right\} .
$$

Assume that we have $m$ samples $N_{1}(\Omega) \ldots N_{m}(\Omega)$ over the same domain $\Omega$, or over domains of the same size $|\Omega|$. We can then use these samples together with the method of moments to estimate the parameters of the prior $\sigma$ and $l$ as follows. Note that,

$$
\begin{aligned}
E[N(\Omega)] & =E[E[N(\Omega) \mid \lambda]] \\
& =E\left[\int_{\Omega} \lambda(s) d s\right] \\
& =\int_{\Omega} E[\lambda(s)] d s \\
& =\int_{\Omega} E\left[\alpha g^{2}(s)\right] d s \\
& =\alpha \int_{\Omega} K(s, s) d s \\
& =\alpha \int_{\Omega} \sigma^{2} d s \\
& =\alpha \sigma^{2}|\Omega| \\
V[N(\Omega)] & =V[E[N(\Omega) \mid \lambda]]+E[V[N(\Omega) \mid \lambda]] \\
& =V[E[N(\Omega) \mid \lambda]]+E\left[\int_{\Omega} \lambda(s) d s\right] \\
& =V[E[N(\Omega) \mid \lambda]]+\alpha \sigma^{2}|\Omega|
\end{aligned}
$$

Further, let $Z=V[E[N(\Omega) \mid \lambda]]$, then

$$
\begin{aligned}
Z & =V\left[\int_{\Omega} \lambda(s) d s\right] \\
& =E\left[\left(\int_{\Omega} \lambda(s) d s\right)^{2}\right]-E^{2}\left[\int_{\Omega} \lambda(s) d s\right] \\
& =E\left[\left(\int_{\Omega} \alpha g^{2}(s) d s\right)^{2}\right]-\left(\alpha \sigma^{2}|\Omega|\right)^{2} \\
& =\alpha^{2} \int_{\Omega} \int_{\Omega} E\left[g^{2}(s) g^{2}(t)\right] d s d t-\left(\alpha \sigma^{2}|\Omega|\right)^{2} \\
& =\alpha^{2} \int_{\Omega} \int_{\Omega} 2 \operatorname{cov}^{2}(g(s), g(t))+v[g(s)] v[g(t)] d s d t \\
& -\left(\alpha \sigma^{2}|\Omega|\right)^{2}
\end{aligned}
$$




$$
\begin{aligned}
& =2 \alpha^{2} \sigma^{4}\left(\int_{s_{1}=0}^{a} \int_{t_{1}=0}^{a} \exp \left\{-\frac{1}{l^{2}}\left(s_{1}-t_{1}\right)^{2}\right\} d s_{1} d t_{1}\right) \\
& \left(\int_{s_{2}=0}^{b} \int_{t_{2}=0}^{b} \exp \left\{-\frac{1}{l^{2}}\left(s_{2}-t_{2}\right)^{2}\right\} d s_{2} d t_{2}\right) \\
& +\alpha^{2} \sigma^{4}|\Omega|^{2}-\left(\alpha \sigma^{2}|\Omega|\right)^{2} \\
& =2 \alpha^{2} \sigma^{4}\left(\sqrt{\pi} a l \sqrt{1-\exp \left\{-\frac{a}{l^{2}}\right\}}\right) \\
& \left(\sqrt{\pi} b l \sqrt{1-\exp \left\{-\frac{b}{l^{2}}\right\}}\right)
\end{aligned}
$$

In the last equation above, if $a$ and $b$ are large and $l$ is small, which are the real case in general, then terms $\sqrt{1-\exp \left\{-\frac{a}{l^{2}}\right\}}$ and $\sqrt{1-\exp \left\{-\frac{b}{l^{2}}\right\}}$ are approximately equal to 1 and we have $Z \approx 2 \alpha^{2} \sigma^{4} \pi a b l^{2}$. Therefore, $V[N(\Omega)]=2 \alpha^{2} \sigma^{4} \pi a b l^{2}+\alpha \sigma^{2}|\Omega|$. Based on the method of moments, we have the following two equations,

$$
\begin{aligned}
\bar{N} & =\frac{1}{m} \sum_{i=1}^{m} N_{i}(\Omega)=\alpha \sigma^{2}|\Omega| \\
S^{2} & =\frac{1}{m} \sum_{i=m}^{m}\left(N_{i}(\Omega)-\bar{N}\right)^{2}=2 \alpha^{2} \sigma^{4} 2 \pi a b l^{2}+\alpha \sigma^{2}|\Omega|
\end{aligned}
$$

Solve for $\sigma$ and $l$ (set $\alpha=1$ ),

$$
\begin{aligned}
\sigma & =\sqrt{\frac{\bar{N}}{a b}} \\
l & =\sqrt{\frac{a b(V[N(\Omega)]-\bar{N})}{2 \pi \bar{N}^{2}}}
\end{aligned}
$$

\subsection{Concavity of the Forward Model}

Recall that $\lambda_{m}=\alpha \phi\left(g_{m}\right)$, with $\alpha>0$. Choosing $p=2$, we obtain for the model in Eq. (3),

$$
\begin{gathered}
\nabla_{g_{m}} \ln p(y \mid g)=-4 \beta\left(g_{m}-\sqrt{y_{m}}\right)^{3} \\
\nabla \nabla_{g_{m}} \ln p(y \mid g)=-12 \beta\left(g_{m}-\sqrt{y_{m}}\right)^{2}
\end{gathered}
$$

Note that the last quantity is continuous and negative so that the matrix $W$ is positive semi-definite.

\section{References}

1. Kaggle competition noaa fisheries steller sea lion population count. https://www.kaggle.com/c/noaa-fisheriessteller-sea-lion-population-count. Accessed: 2017-04-28

2. Arteta, C., Lempitsky, V., Noble, J.A., Zisserman, A.: Interactive object counting. In: European Conference on Computer Vision, pp. 504-518. Springer (2014)
3. Chan, A.B., Liang, Z.S.J., Vasconcelos, N.: Privacy preserving crowd monitoring: Counting people without people models or tracking. In: Computer Vision and Pattern Recognition, 2008. CVPR 2008. IEEE Conference on, pp. 1-7. IEEE (2008)

4. Cho, S.Y., Chow, T.W., Leung, C.T.: A neural-based crowd estimation by hybrid global learning algorithm. IEEE Transactions on Systems, Man, and Cybernetics, Part B (Cybernetics) 29(4), 535-541 (1999)

5. Cox, D.R.: Some statistical methods connected with series of events. Journal of the Royal Statistical Society. Series B (Methodological) pp. 129-164 (1955)

6. Dalal, N., Triggs, B.: Histograms of oriented gradients for human detection. In: Computer Vision and Pattern Recognition, 2005. CVPR 2005. IEEE Computer Society Conference on, vol. 1, pp. 886-893. IEEE (2005)

7. Dassios, A., Jang, J.W.: Pricing of catastrophe reinsurance and derivatives using the cox process with shot noise intensity. Finance and Stochastics 7(1), 73-95 (2003)

8. Deng, J., Berg, A., Satheesh, S., Su, H., Khosla, A., FeiFei, L.: Ilsvrc-2012 (2012)

9. Everingham, M., Van Gool, L., Williams, C.K., Winn, J., Zisserman, A.: The pascal visual object classes (voc) challenge. International journal of computer vision $\mathbf{8 8}(2)$, 303-338 (2010)

10. Felzenszwalb, P.F., Girshick, R.B., McAllester, D. Ramanan, D.: Object detection with discriminatively trained part-based models. IEEE transactions on pattern analysis and machine intelligence 32(9), 1627-1645 (2010)

11. Fiaschi, L., Koethe, U., Nair, R., Hamprecht, F.A.: Learning to count with regression forest and structured labels. In: Pattern Recognition (ICPR), 2012 21st International Conference on, pp. 2685-2688. IEEE (2012)

12. Flaxman, S., Wilson, A.G., Neill, D.B., Nickisch, H., Smola, A.J.: Fast kronecker inference in gaussian processes with non-gaussian likelihoods. In: International Conference on Machine Learning, vol. 2015 (2015)

13. Ge, W., Collins, R.T.: Marked point processes for crowd counting. In: Computer Vision and Pattern Recognition, 2009. CVPR 2009. IEEE Conference on, pp. 2913-2920. IEEE (2009)

14. Girshick, R.: Fast r-cnn. In: Proceedings of the IEEE International Conference on Computer Vision, pp. 14401448 (2015)

15. Girshick, R., Donahue, J., Darrell, T., Malik, J.: Rich feature hierarchies for accurate object detection and semantic segmentation. In: Proceedings of the IEEE conference on computer vision and pattern recognition, pp. 580-587 (2014)

16. Han, W., Rajan, P., Frazier, P.I., Jedynak, B.M.: Bayesian group testing under sum observations: A parallelizable two-approximation for entropy loss. IEEE Transactions on Information Theory 63(2), 915-933 (2017)

17. He, K., Zhang, X., Ren, S., Sun, J.: Spatial pyramid pooling in deep convolutional networks for visual recognition. In: European Conference on Computer Vision, pp. 346361. Springer (2014)

18. Idrees, H., Saleemi, I., Seibert, C., Shah, M.: Multi-source multi-scale counting in extremely dense crowd images. In: Proceedings of the IEEE Conference on Computer Vision and Pattern Recognition, pp. 2547-2554 (2013)

19. Kingman, J.F.C.: Poisson processes. Wiley Online Library (1993)

20. Kong, D., Gray, D., Tao, H.: A viewpoint invariant approach for crowd counting. In: Pattern Recognition, 2006. 
ICPR 2006. 18th International Conference on, vol. 3, pp. 1187-1190. IEEE (2006)

21. Krizhevsky, A., Sutskever, I., Hinton, G.E.: Imagenet classification with deep convolutional neural networks. In: Advances in neural information processing systems, pp. 1097-1105 (2012)

22. Lafarge, F., Descombes, X., et al.: Geometric feature extraction by a multimarked point process. IEEE transactions on pattern analysis and machine intelligence $\mathbf{3 2}(9)$, 1597-1609 (2010)

23. Lempitsky, V., Zisserman, A.: Learning to count objects in images. In: Advances in Neural Information Processing Systems, pp. 1324-1332 (2010)

24. Lin, T.Y., Maire, M., Belongie, S., Hays, J., Perona, P., Ramanan, D., Dollár, P., Zitnick, C.L.: Microsoft coco: Common objects in context. In: European Conference on Computer Vision, pp. 740-755. Springer (2014)

25. Liu, X., Tu, P.H., Rittscher, J., Perera, A., Krahnstoever, N.: Detecting and counting people in surveillance applications. In: Advanced Video and Signal Based Surveillance, 2005. AVSS 2005. IEEE Conference on, pp. 306311. IEEE (2005)

26. Lowe, D.G.: Distinctive image features from scaleinvariant keypoints. International journal of computer vision 60(2), 91-110 (2004)

27. MacKay, D.J.: Information theory, inference and learning algorithms. Cambridge university press (2003)

28. Marana, A., Velastin, S., Costa, L., Lotufo, R.: Estimation of crowd density using image processing. In: Image Processing for Security Applications (Digest No.: 1997/074), IEE Colloquium on, pp. 11-1. IET (1997)

29. Merchan-Perez, A., Rodriguez, J., Alonso-Nanclares, L., Schertel, A., DeFelipe, J.: Counting synapses using fib/sem microscopy: A true revolution for ultrastructural volume reconstruction. Frontiers in Neuroanatomy 3(18) (2009)

30. Moghaddam, B., Pentland, A.: Probabilistic visual learning for object representation. IEEE Transactions on pattern analysis and machine intelligence 19(7), 696-710 (1997)

31. Onoro-Rubio, D., López-Sastre, R.J.: Towards perspective-free object counting with deep learning. In: European Conference on Computer Vision, pp. 615-629. Springer (2016)

32. Pham, T.T., Chin, T.J., Schindler, K., Suter, D.: Interacting geometric priors for robust multimodel fitting. IEEE transactions on image processing 23(10), 46014610 (2014)

33. Pham, T.T., Hamid Rezatofighi, S., Reid, I., Chin, T.J.: Efficient point process inference for large-scale object detection. In: Proceedings of the IEEE Conference on Computer Vision and Pattern Recognition, pp. 2837-2845 (2016)

34. Rajan, P., Han, W., Sznitman, R., Frazier, P., Jedynak, B.: Bayesian multiple target localization. In: International Conference on Machine Learning, pp. 1945-1953 (2015)

35. Rasmussen, C.E.: Gaussian processes for machine learning (2006)

36. Redmon, J., Divvala, S., Girshick, R., Farhadi, A.: You only look once: Unified, real-time object detection. In: Proceedings of the IEEE Conference on Computer Vision and Pattern Recognition, pp. 779-788 (2016)

37. Redmon, J., Farhadi, A.: Yolo9000: better, faster, stronger. arXiv preprint arXiv:1612.08242 (2016)

38. Ren, S., He, K., Girshick, R., Sun, J.: Faster r-cnn: Towards real-time object detection with region proposal networks. In: Advances in neural information processing systems, pp. 91-99 (2015)

39. Ross, S.M.: Stochastic processes. 1996 (1996)

40. Saatçi, Y.: Scalable inference for structured gaussian process models. Ph.D. thesis, Citeseer (2012)

41. Sam, D.B., Surya, S., Babu, R.V.: Switching convolutional neural network for crowd counting. In: Proceedings of the IEEE Conference on Computer Vision and Pattern Recognition, vol. 1, p. 6 (2017)

42. Simonyan, K., Zisserman, A.: Very deep convolutional networks for large-scale image recognition. arXiv preprint arXiv:1409.1556 (2014)

43. Sindagi, V.A., Patel, V.M.: Cnn-based cascaded multitask learning of high-level prior and density estimation for crowd counting. In: Advanced Video and Signal Based Surveillance (AVSS), 2017 14th IEEE International Conference on, pp. 1-6. IEEE (2017)

44. Sindagi, V.A., Patel, V.M.: A survey of recent advances in cnn-based single image crowd counting and density estimation. Pattern Recognition Letters (2017)

45. Uijlings, J.R.R., van de Sande, K.E.A., Gevers, T., Smeulders, A.W.M.: Selective Search for Object Recognition. International Journal of Computer Vision 104(2), 154-171 (2013). DOI 10.1007/s11263-013-0620-5

46. Verdie, Y., Lafarge, F.: Detecting parametric objects in large scenes by monte carlo sampling. International Journal of Computer Vision 106(1), 57-75 (2014)

47. Walach, E., Wolf, L.: Learning to count with cnn boosting. In: European Conference on Computer Vision, pp. 660-676. Springer (2016)

48. Wang, C., Zhang, H., Yang, L., Liu, S., Cao, X.: Deep people counting in extremely dense crowds. In: Proceedings of the 23rd ACM international conference on Multimedia, pp. 1299-1302. ACM (2015)

49. Warren, S., Hewett, P., Foltz, C.: The kx method for producing k-band flux-limited samples of quasars. Monthly Notices of the Royal Astronomical Society 312(4), 827$832(2000)$ 